\title{
Hydrothermal synthesis of bismuth ferrite nanostructures for supercapacitor
}

\author{
N. Chakrabarty and A.K. Chakraborty* \\ Department of Physics, NIT Durgapur, Durgapur-713209, India \\ Centre of Excellence in Advanced Materials, NIT Durgapur, Durgapur-713209, India \\ *Email: amitkc61@gmail.com
}

In the past decade, a lot of research has been focussed on supercapacitors as energy storage device. Transition metal oxides like $\mathrm{NiO}, \mathrm{Co}_{3} \mathrm{O}_{4}$, $\mathrm{MnO}_{2}$ and their composites have been exploited in this field for their large natural abundance and superior redox activity. Binary transition metal oxides (BTMOs), containing at least one transition metal ion and one or more electrochemically active/inactive ions offer rich redox reaction, good electrical conductivity, wide potential window, large number of active sites and high stability due to synergistic influence of the components [1].

Here we report the synthesis of bismuth ferrite $\left(\mathrm{BiFeO}_{3}\right.$, hereafter called $\left.\mathrm{BFO}\right)$ nanostructures using nitrate precursors of bismuth and iron following a hydrothermal route as both bismuth and iron exhibit different oxidation states resulting in more active sites for redox reaction. The reaction product was filtered and calcined in air to effect the phase transformation of $\mathrm{BFO}$ nanostructures. The microstructural and compositional analyses were carried out using powder X-ray diffraction (XRD), high resolution transmission electron microscopy (HRTEM) and energy dispersive analysis by x-rays (EDAX). Further, the electrochemical properties of the nanocomposite were investigated by cyclic voltammetry $(\mathrm{CV})$, chronopotentiometry $(\mathrm{CP})$ and electrochemical impedance spectroscopy (EIS) in order to evaluate the potential of BFO nanostructures as electrode materials for supercapacitors.

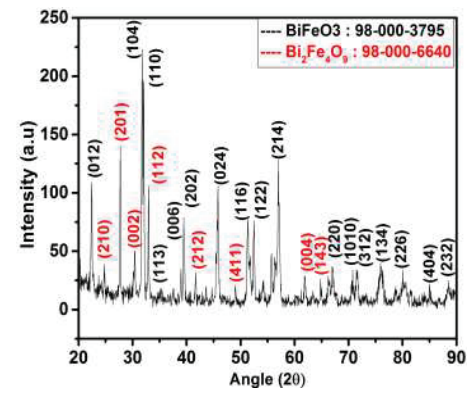

Figure 1: X-ray diffractogram of BFO nanostructures

The XRD plot of BFO nanostructures (Figure 1) shows peaks corresponding to both BFO and $\mathrm{Bi}_{2} \mathrm{Fe}_{4} \mathrm{O}_{9}$ (as indicated in red) in accordance with JCPDS card nos. 98-000-3795 and 98-000-6640, respectively. It is obvious that the synthesis process adopted here resulted in the formation of the mixed phases of bismuth ferrite.

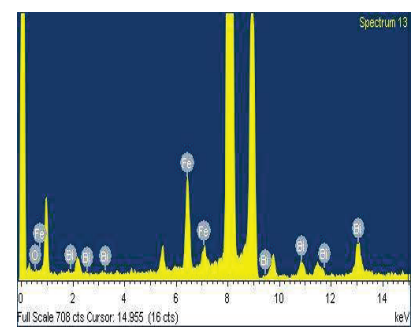

Figure 2: EDAX spectrum of BFO

The EDAX spectrum (Figure 2) of the BFO shows the presence of bismuth, iron and oxygen signal as expected. HRTEM images (Figure 3) shows agglomerated structures with strong interconnection, which is a common feature for ferrite materials. The calculated average particle size of BFO is found to range between 8 and $27 \mathrm{~nm}$.
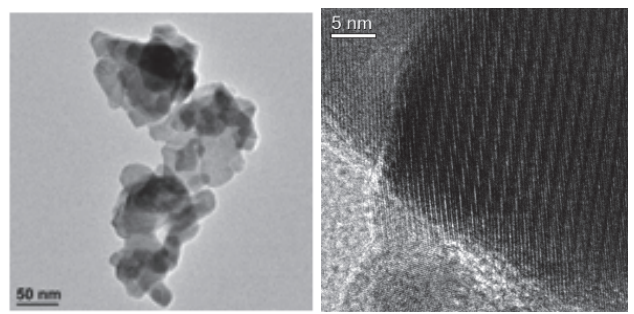

Figure 3: HRTEM images of BFO nanostructures. Moiré pattern can also be seen in the right image

The electrochemical studies performed using a three electrode system with BFO film deposited on a glassy carbon working electrode revealed good specific capacitance values. While calculations from $\mathrm{CV}$ curves gave a value of $169 \mathrm{~F} / \mathrm{g}$ at $5 \mathrm{mV} / \mathrm{s}, \mathrm{CP}$ curves resulted $157 \mathrm{~F} / \mathrm{g}$ at $1 \mathrm{~A} / \mathrm{g}$ which are higher than the previously reported specific capacitance value $(81 \mathrm{~F} / \mathrm{g})$ for $\mathrm{BFO}[2]$ indicating the suitability of BFO as electrode material in supercapacitor applications.

\section{References}

1. M.V. Reddy, G.V.SubbaRao, B.V.R. Chowdari, Chem. Rev. 113 (2013) 5364.

2. C.D. Lokhande, T.P.Gujar, V.R. Shinde, R.S.Mane, S.H. Han, Electrochem. Commun. 9(2007)1805-1809. 